Documentation et bibliothèques

DOCUMENTATION BIBLIOTHEQUES

\title{
Les normes de bibliothèques de collège : le Québec rattrapera-t-il le pas?
}

\section{Angèle Murphy}

Volume 19, numéro 4, décembre 1973

URI : https://id.erudit.org/iderudit/1055738ar

DOI : https://doi.org/10.7202/1055738ar

Aller au sommaire du numéro

\section{Éditeur(s)}

Association pour l'avancement des sciences et des techniques de la documentation (ASTED)

\section{ISSN}

0315-2340 (imprimé)

2291-8949 (numérique)

Découvrir la revue

\section{Citer cet article}

Murphy, A. (1973). Les normes de bibliothèques de collège : le Québec rattrapera-t-il le pas ? Documentation et bibliothèques, 19(4), 161-164. https://doi.org/10.7202/1055738ar
Résumé de l'article

Un projet de normes québécoises pour les bibliothèques de collège est analysé en regard des normes américaines et canadiennes.

L'auteur reconnaît la valeur pratique de la première édition des normes québécoises tout en admettant la supériorité scientifique des normes américaines.
Tous droits réservés (C) Association pour l'avancement des sciences et des techniques de la documentation (ASTED), 1973
Ce document est protégé par la loi sur le droit d'auteur. L'utilisation des services d'Érudit (y compris la reproduction) est assujettie à sa politique d'utilisation que vous pouvez consulter en ligne.

https://apropos.erudit.org/fr/usagers/politique-dutilisation/ 
Les normes de bibliothèques de collège: Le Québec rattrapera-t-il le pas?

Angèle Murphy

Coordonnateur du Centre de documentation Service général des moyens d'enseignement Ministère de l'Éducation

Montréal

Un projet de normes québécoises pour les bibliothèques de collège est analysé en regard des normes américaines et canadiennes.

L'auteur reconnait la valeur pratique de la première édition des normes québécoises tout en admettant la supériorité scientifique des normes américaines.

Dans un article de Jean-Rémi Brault intitulé: "Le rapport Parent... cinq ans après", on pouvait lire:

"...ceux (signifiant les responsables du rapport Parent) qui voulaient ainsi rendre à la bibliothèque sa vraie vocation, ceux-là comprenaient-ils toutes les implications d'une telle entreprise?"1

Nous nous demandons aujourd'hui si l'aventure cégépienne s'avère, jusqu'à maintenant, avantageuse pour les bibliothèques de collège? Nous rappelons que le système de répartition budgétaire s'établit actuellement selon le nombre d'étudiants inscrits (per capita). Ce qui nous amène à une première observation: les bibliothèques de collèges importants, soient les collèges de 3,000 à 5,000 étudiants, semblent s'organiser convenablement. Par ailleurs, plus ces collèges ont une bibliothèque intégrée à la pédagogie, plus les collections sont multi-media, plus les usagers augmentent et plus les lacunes en personnel et en services deviennent aiguës. Que dire alors des collèges de 1,000 et 2,000 étudiants? Les directeurs de bibliothèques semblent désarmés, parfois même désespérés. On se trouve souvent devant un budget d'acquisitions de $\$ 3,000$ par an-

1. Jean-Rémi Brault, "Le rapport Parent. . . cinq ans après", Bulletin de l'ACBLF, vol. 17, no 2 (juin 1971), 81. née. On est même obligé de remercier du personnel et d'abandonner certains services.

De son côté, la DIGEC (Direction générale de l'enseignement collégial) semble bien peu sensibilisée à la situation précaire des bibliothèques de collège. Bien sûr, on invoque là-haut la rationalisation des budgets et les coupures drastiques infligées par le Conseil du trésor au niveau collégial. Toutefois, on admet que la planification budgétaire (PPBS) est systémique, mais au niveau de l'analyse quantitative seulement et non au niveau de l'analyse qualitative. C'est donc admettre que l'analyse des besoins du milieu se fait de façon fort arbitraire. A partir de quels critères les autorités gouvernementales accordent-elles des budgets aux services de bibliothèques et de techniques audiovisuelles, lesquels sont regroupés dans l'enveloppe IV?

Les normes d'excellence qui inspirent le ministère de l'Éducation sont assurément désuètes puisqu'elles offrent $\$ 65.00$ per capita, plus $\$ 25,000$ de base pour 197374. Est-ce à dire que le Québec ne possède aucune norme officielle pour les bibliothèques de collège? C'est, en effet, le cas.

Au Québec, on pensa, en 1970, que des normes adaptées étaient absolument nécessaires pour les bibliothèques de collège. On voulait d'abord créer un instrument qui servirait aux directeurs de bibliothèques. De plus, ces normes seraient utiles à la DIGEC pour établir les critères de base servant à la répartition des budgets.

Gilles Grondin écrivait, dans une lettre d'invitation aux directeurs de bibliothèques:

"Le but de cette étude est de proposer au ministère de l'Éducation des normes précises sur des questions aussi spécifiques que le personnel, le nombre de volumes, etc."

Ce travail sur les normes a donc été conçu par l'exécutif de la Sous-commission des directeurs de bibliothèques de la Fédération des Cegep, avec la collaboration directe de tous leurs confrères. Les normes ont été discutées et approuvées au cours de deux assemblées générales convoquées à cette fin. Toutefois, le rapport n'a jamais été adopté officiellement. Et depuis, il est devenu périmé à bien des égards. Il s'agissait d'une première expérience: on voulait présenter à la fois une orientation et certaines normes quantitatives. Cependant, plusieurs situations ont évolué et bien des points sont à redéfinir. 
Or, en décembre 1972, une enquête téléphonique auprès de douze (12) directeurs de bibliothèques a révélé que ceux-ci souhaitaient, à l'unanimité, la révision en profondeur des normes et sa présentation aux autorités concernés. Une résolution fut formulée comme suit:

"Nous recommandons que l'exécutif 1972-73 de la Sous-commission des directeurs de bibliothèques procède à une révision des normes rédigées en 1970.

Que la Sous-commission des directeurs de bibliothèques s'assure de la mise à jour annuelle de ces normes."

Depuis 1971, les premières normes dormaient sur les tablettes; on n'en parlait qu'en coulisses. Mais lors de l'assemblée générale des directeurs de bibliothèques de février 1973, on donnait au nouvel exécutif le mandat de procéder à une révision complète des normes de 1971 et de présenter un nouveau projet à l'automne 1973.

L'assemblée générale des directeurs de bibliothèques de collège, tenue en novembre dernier, nous a permis d'en étudier le texte provisoire. Nous dégagerons donc, au cours de cette analyse, les principales caractéristiques des futures normes québécoises, en les comparant aux normes américaines et canadiennes.

\section{Les normes américaines}

Les dernières normes américaines et canadiennes concernant les bibliothèques de collège ont été publiées en 1972.

Une approche qualitative des normes prévaut chez les Américains. On discerne, en effet, tout au long du document, une préoccupation d'analyse scientifique, d'approche systémique (systems approach). Ces normes ont été formulées conjointement par l'American Association of Junior Colleges et l'Association of College and Research Libraries. Approuvées officiellement en janvier 1972, elles seront révisées annuellement.

Dans leur analyse qualitative, les Américains tiennent compte du fait que les objectifs des institutions peuvent varier et que le nombre d'étudiants des collèges est un facteur important. Les recommandations ont été formulées à la suite d'une consultation auprès de professionnels compétents et après un essai jugé concluant dans les meilleures institutions ayant servi à l'auto-évaluation et à la projection de planification. On suggère aux institutions d'appliquer les différents critères en fonc- tion des buts, des objectifs et de la clientèle de chacune. Les normes américaines sont donc particulièrement utiles pour donner une orientation, une philosophie du "Learning Resource Center".

Les institutions auront des collections et des services proportionnels quantitativement aux objectifs qu'elles se seront fixés.

La ligne de pensée perçue dans ces normes fait réaliser l'importance de tenter une analyse rationnelle et éclairée des bésoins. On vise, aux États-Unis, à l'organisation du Library Learning Resource Center qui devient une unité administrative intégrant les ressources imprimées et audiovisuelles ainsi que l'équipement et les services permettant leur utilisation.

Ce service, tel que décrit, devra répondre à la pédagogie de l'institution. Le collège désire l'exploration du savoir par la participation active en classe et par le travail individuel. Le Centre permet l'utilisation des ressources dans le but d'atteindre des objectifs pédagogiques opérationnels et pré-définis: "The L.R.C. expresses the educational philosophy of the institution it serves". ${ }^{2}$ De ce fait, il devient essentiel de définir les besoins individuels, les besoins du programme et les besoins de l'institution.

\section{Les normes canadiennes}

L'Association canadienne des bibliothèques de collège et d'université (ACBCU/ CACUL) a aussi réalisé qu'on ne fixait pas des institutions dans des normes quantitatives. Des normes basées, au surplus, sur quels critères? Mais il arrive que le processus de création utilisé pour les normes canadiennes est beaucoup moins scientifique. On a, en effet, travaillé pendant un an et demi en réalisant des analyses en équipes et en consultant le milieu. Une comparaison avec les normes existantes américaines et québécoises a également été tentée. Les normes canadiennes sont donc le résultat d'un consensus découlant de consultations générales. II s'agit davantage d'une compilation des réponses à une enquête que d'une réelle recherche scientifique. Nous y retrouvons surtout des normes qualitatives couvrant les sujets suivants: administration et organisation, budget, construction, collections, services et personnel. Les seules normes quantitatives incluses concernent le budget, les col-

2. Association of College and Research Libraries, "AAJC-ACRL Guidelines for Two-year College Library Learning Resource Centers", College and Research Libraries News, no. 9 (October 1971), 267. 
lections, le nombre de places et le personnel. On a avoué que ces critères quantitatifs n'avaient été établis qu'à titre de suggestions minimales et afin de faciliter les prévisions budgétaires.

D'un point de vue analytique, il existe deux différences fondamentales avec les normes américaines:

- Le statut du directeur de la bibliothèque est inférieur en ce sens qu'il a moins de pouvoir: "The final responsability for the library belongs with the college's chief administrator and the college's governing board or council" 3 .

-On ne suggère pas une structure intégrée qui serait l'équivalent du "Learning Resource Center" américain. muns:

Certains points sont cependant com-

- La bibliothèque est sous la juridiction des services pédagogiques.

- Les professionnels sont des membres actifs et informés des politiques, des procédures et des décisions concernant le personnel.

-Des modalités de participation doivent exister au plan des politiques et des procédures générales ainsi que des décisions concernant le personnel.

\section{Les normes québécoises}

Dans l'avant-propos des normes québécoises, on lit que le texte "constitue un document de base pour le développement et l'évaluation des bibliothèques" 4 . Nous comprenons au départ que le texte analysé ne soit pas le texte final adopté par les membres. Cependant, on nous a assurés que les modifications ne pouvaient pas être majeures, car on s'attend à une adoption globale du projet.

Nous pouvons donc affirmer, d'ores et déjà, que les normes québécoises de 1973 ne sont pas une refonte complète des normes de 1971. En effet, un représentant du comité de rédaction a expliqué qu'on s'était appliqué à simplifier le texte et son contenu. Ainsi disparaît tout le passage sur "le rôle des principaux services". On a compris que cela relevait davantage de la régie interne ou d'un manuel de bibliothéconomie.

3. Canadian Association of College and University Libraries/Association canadienne des bibliothèques de collège et d'université. Standards Recommended for Canadian Community College Libraries, Vancouver, 1972 , p. 5

4. Fédération des Cegep. Commission des directeurs de bibliothèques. Normes pour les bibliothèques de Cegep. Document de travail, 1973, p. 1, article 0.1.
On a voulu aussi adapter davantage certains points qui font maintenant partie des moeurs et qui n'ont plus à être prouvés. C'est ainsi que sont disparus les services parallèles de la documentation imprimée et audio-visuelle. On présente carrément une structure d'intégration des media. Les services publics intègrent la documentation textuelle et non-textuelle.

D'autre part, n'ayant pas effectué d'étude plus appronfondie, il ne faut pas s'attendre à une modification majeure du texte de 1971. Cependant, il eut été intéressant que les rédacteurs précisent davantage le statut du directeur de bibliothèque comme personne-cadre dans les services pédagogiques, et le rôle des bibliothécaires professionnels comme participants à la pédagogie de l'institution.

Le chapitre intitulé: "Structure et administration" semble faible sur le plan de l'argumentation. II aurait fallu se référer davantage aux deux rapports présentés à l'ACBLF portant l'un sur le statut du directeur de bibliothèque et l'autre sur le statut des bibliothécaires professionnels. II faut se rappeler que les administrateurs et les fonctionnaires ont des désirs de centralisation et des soucis d'économie. C'est pourquoi l'argument administratif devrait être plus étoffé pour motiver les structures et les fonctions.

De plus, le chapitre traitant de la documentation n'est pas suffisamment exploité. Contrairement aux normes américaines, on dit peu concernant les politiques écrites en matière de sélection ou de choix documentaire. II n'est pas fait mention non plus de politique d'exemplaires multiples, compte tenu des besoins accrus résultant du travail individuel ou en groupes. On n'y discute pas du niveau intellectuel des collections, ni de la particularité des différents media. Ce chapitre doit, de toute évidence, être approfondi. II faut aider les législateurs à se souvenir que le prix d'un film 8mm n'est pas l'équivalent d'un "Que sais-je?" II faut aussi sensibiliser le milieu au fait que l'étudiant du niveau collégial, ayant un apprentissage de plus en plus individualisé, doit accéder facilement à la documentation nécessaire. Les collections doivent donc être variées et les exemplaires multiples. On remarque avec plaisir que la norme suggérée de 50,000 documents écrits et de 50,000 documents audio-visuels est réaliste et assez conforme aux besoins des collèges. Il est illusoire de croire que 125,000 titres de documents écrits sont utilisés et exploités au niveau collégial. 


\section{Conclusion}

En somme, le projet des normes de 1973 présente des visées réalistes, peut-être trop simplifiées mais respectant l'idéologie du groupe de travail de 1971. Les titres des chapitres sont d'ailleurs les mêmes: 1) nature de la bibliothèque de collège; 2 ) structure et administration; 3) personnel; 4) documentation; 5) budget; 6) locaux, mobilier et équipement. Nous ne pouvons plus croire aujourd'hui que les meilleures normes sont celles qui offrent les critères quantitatifs les plus élevés. L'approche systémique d'analyse des besoins conçue par les Américains est maintenant appliquée au niveau économique, particulièrement au niveau de la planification budgétaire. On peut y déceler une méthode de travail plus objective et plus scientifique. Sous l'influence de ces méthodes, l'American Association of Junior Colleges et l'Association of College and Research Libraries ont conjointement formulé des normes qui se veulent une orientation, un esprit qui permettra aux bibliothèques de collège d'atteindre un niveau d'excellence en accord avec leurs besoins véritables.

Les normes mises de l'avant au Québec par le groupe des directeurs de bibliothèques de la Fédération des Cegep, auront probablement la chance d'être endossées par les directeurs des services pédagogiques et les directeurs généraux. Peut-être ensuite seront-elles consultées par la DIGEC. Nous devons surtout espérer qu'elles orienteront les objectifs et les activités de nos bibliothèques de collège. Ainsi, progressivement, tous les intéressés sentiront le besoin de produire des normes qui ne soient plus qu'un document de base et des normes minimales, mais de véritables normes d'excellence. II reste que la première édition des normes québécoises pour les bibliothèques de collège était attendue depuis Iongtemps. Elle aura le mérite d'avoir rassemblé les réflexions de plusieurs bibliothécaires soucieux de l'avancement des bibliothèques de collège. Et surtout, elle aura le mérite de pouvoir orienter en esprit, et en fait, les décisions concernant les bibliothèques et le milieu collégial•

\section{Autres sources consultées}

American Library Association and National Education Association. Standards for School Media Programs. Chicago and Washington.

Association canadienne des bibliothécaires de langue française. Section des collèges. Comité d'étude sur le statut et les conditions salariales des directeurs de bibliothèques de Cegep. Rapport. Montréal, 1973.

- Comité d'étude sur le statut et les échelles de traitement du bibliothécaire de Cegep. Rapport. Montréal, 1973.

Blanch, Lloyd E. Accreditation in Higher Education. U.S. Department of Health, Education and Welfare. Washington, U.S. Government Printing Office, 1959. Clift, David H. "Library Associations in the United States and British Commonwealth", Library Trends, vol. 3 , no. 3 (January 1955).

Directeurs de bibliothèques rattachés au CADRE, ACQ, AIES, CEGEP. Assemblée générale conjointe, 3e, Montréal, 1971. Rapport. Montréal, 1971.

Lyle, Guy R. "Evaluation of the College Library" in The administration of the College Library, 3rd Ed., New York, H.W. Wilson Co., 1961.

Oboler, Eli et al. College and University Library ACcreditation Standards. 1957. Chicago, ACRL, 1958 (ACRL monograph, no. 20). 